\title{
Deficiencia de hierro y enriquecimiento de alimentos
}

\author{
DRES: : ABRAHAM STEKEL *, MANUEL OLIYARES *, DRA.: INES LOPEZ ** T. M. PATRICIA CHA- \\ DUD* y T, M. GLORIA CASTANO*.
}

La alta prevalencia de la carencia de hierro ha sido bien documentada en diferentes regiones baciendo de ésta, tal vez, la deficiencia nutricional más difundida en el mundo. En todos los países, los lactantes entre los 6 y 18 meses de edad y las mujeres embarazadas aparecen como los grupos más vulnerables (1). En estudios patrocinados por la OMS en diferentes partes del mundo, el porcentaje de mujeres embarazadas con anemia osciló entre $21 \%$ y $80 \%$, y el porcentaje con deficiencia de hierro fue más alto aún $(40 \%$ a $99 \%)$ usando la saturación de la transferrina bajo $15 \%$ como criterio de deficiencia (2). En niños, en estudios en Sudamérica el porcentaje con anemia ha variado entre un $15 \%$ y un $50 \%$ (1). En Chile, sólo existen datos de prevalencia en lactantes que muestran un porcentaje de anemia que varía entre un $15 \%$ y un $40 \%$ de los casos y un porcentaje de hasta $83 \%$ con saturación de la transferrina bajo $15 \%(3,4,5)$.

$\mathrm{Si}$ bien las consecuencias desfavorables de una anemia severa son evidentes, se ha debatido acerca de las desventajas que tendría una deficiencia de hierro más larvada, llegando incluso a enumerarse posibles ventajas derivadas de esta condición (6). Ultimamente, sin embargo, han comenzado a documentarse efectos antes no reconocidos y posiblemente independientes de la anemia que van desde la disminución en la capacidad de trabajo físico $y$ en el rendimiento intelectual $(7,8)$ hasta una alteración de la inmunidad celular (9) y una mayor frecuencia de infecciones (10).

Resulta evidente que ante la magnitud que al-

\footnotetext{
* Departamento de Nuirición y Tecnología de los Alimentos Sede Santiago Sur, Universidad de Chile.

* Laboratorio de Hematologla. Hospital Arriarán.

Financiado por Grant de la Fundación Ford.
}

canza la deficiencia nutricional de hierro, los esfuerzos deban encaminarse hacia la prevención. En el presente trabajo nos referiremos a las posibilidades de prevención con especial énfasis al problema en 1a infancia.

La prevención de la carencia nutricional de hierro es, en teoría, sencilla. Se necesita conocer cuáles son los grupos expuestos, cuáles son sus requerimientos de hierro y cuál es la forma de llenar estos requerimientos. En la práctica, sin embargo, aunque los grupos expuestos y sus requerimientos han logrado precisarse bastante bien, persisten varios problemas para conseguir una ingesta de hierro adecuada para Ilenar los requerimientos de todos los individuos.

Requerimientos de hierro: el cálculo de los requerimientos se basa en estimaciones de las perrdidas fisiológicas y en las necesidades derivadas del crecimiento. Para un lactante de 1 año de edad se han estimado las pérdidas fisiológicas en $0,4-0,5 \mathrm{mg}$. al dia (1). Las necesidades derivadas del crecimiento por otra parte dependen de la expansión de la masa de hemoglobina. Se sabe que un niño de término normal no aumenta su contenido total de hierro en los primeros cuatro meses ya que durante este período existe una redistribución hacia los tejidos del hierro proveniente de la disminución fisiológica de la masa de hemoglobina. Si se asume a partir del $4^{\circ}$ mes un aumento lineal del contenido total de hierro del organismo en relación con el peso, el incremento diario entre los 4 y los 12 meses sería de $0,5 \mathrm{mg}$. (1). Como las pérdidas, según se vio, son también del orden de $0,5 \mathrm{mg} . / \mathrm{d}$. el total de hierro absorbido que se requiere en este período sería de $1,0 \mathrm{mg}$. al día. El problema se complica, sin embargo, porque la absorción de hierro depende de numerosos 
T A BL A I

RECOMENDACION INGESTA DIARIA DE HIERRO(*)

\begin{tabular}{|c|c|c|c|c|}
\hline & $\begin{array}{c}\text { Hierro Absorbido } \\
\text { Requerido (mg) }\end{array}$ & Ingesta $R \boldsymbol{R}$ & omendada según & tipo de Dieta \\
\hline & & $\left|\begin{array}{c}\text { Alintentos } \\
\text { animales } \\
\text { bajo Jo\% de } \\
\text { las calorías }(\mathrm{mg})\end{array}\right|$ & $\begin{array}{c}\text { Alimentos } \\
\text { animales } \\
\text { bajo } 10-25 \% \\
\text { de las calorias } \\
\text { (mg) }\end{array}$ & $\begin{array}{l}\text { Alimentos } \\
\text { animales } \\
\text { de las calorias } \\
\text { sobre } 25 \% \\
\text { (mg) }\end{array}$ \\
\hline Lactantes $0-4$ meses & 0,5 & a & a & a \\
\hline $5-12$ meses & 1,0 & 10 & 7 & 5 \\
\hline $1-12$ años & 1,0 & 10 & 7 & 5 \\
\hline Hombres $13-16$ años & 1,8 & 18 & 12 & 9 \\
\hline Mujeres $13-16$ años & 2,4 & 24 & 18 & 12 \\
\hline Mujeres Menstruantes b & 2,8 & $2 B$ & 19 & 14 \\
\hline Hombres Adultos & 0,9 & 9 & 6 & 5 \\
\hline
\end{tabular}

a: Alimentación al Pecho, se asume es adecuadás.

b: Para Mujeres no menstruantes, la ingesta fecomendada es la misma que para el hombre.

*: FAO/WHO, 1970.

factores, en especial de la composición de Ja dieta. La Tabla I muestra las ingestas diarias recomendadas por la FAO/WHO (1). Las recomendaciones para los lactantes han sido posteriormente revisadas (2) siendo rebajadas en aproximadamente un $30 \%$.

Absorción de hierro de los alimentos: la cantidad de hierro absorbida por el organismo depende de varios factores tales como la cantidad total de hierro presente en la dieta, lo disponible que sea este hierro para la absorción y la regulación del proceso de absorción por parte del organismo (11). En los últimos años ha habido un progreso importante en el conocimiento de la absorción de hierro, y en especial del hierro de los alimentos. Este progreso fue posible gracias a la introducción de técnicas de absorción insotópicas en que el hierro radioactivo era incorporado biológicamente a los alimentos (12). Estudios recientes de Layrisse y colaboradores (Fig. 1) empleando esta técnica en 524 personas adultas (la mitad deficientes en hierro), han indicado promedios de absorción de hierro de alimentos vegetales que varían entre cl $1 \%$ para el arroz y las espinacas y el $6 \%$ para la soya, con valores intermedios de $3 \%$ para el maíz y los porotos negros, $4 \%$ para las lechugas y $5 \%$ para el trigo (13). La absorción promedio del hierro de alimentos de origen animal osciló entre un $7 \%$ para la ferritina y un $22 \%$ para el músculo de ternera con valores intermedios de $11 \%$ para el pescado, $12 \%$ para la hemoglobina y $13 \%$ para el hígado.

Se ha demostrado también que existe interacción entre los alimentos. Es así como la carne au- menta la absorción del hierro del maíz y los porotos y la administración de ácido ascórbico aumenta la ábsorción del hierro alimentario no hemínico, en tanto que el huevo y los fitatos producen una marcada depresión (2). En el caso de sales de hierro agregadas como suplemento a alimentos, aunque la información es limitada, se puede decir que la absorción se modifica en primer lugar al mezclar el suplemento con el alimento determinado. En ese momento la absorción se hace comparable a la absorción del hierro intrínseco de ese alimento. Luego al mezclar el alimento enriquecido con otros alimentos, la absorción del hierro suplementario se modifica de acuerdo a la composición de la dieta total (14).

Prevención de la carencia de hierro en la infancia: el ideal de prevención de una deficiencia nutricional es a través del consumo de una dieta adecuada. Este ideal, sin embargo, en el caso del hierro es difícil de alcanzar en todos los individuos, ya sea porque la dieta habitual es pobre en hierro o porque el hierro de esa dieta se absorbe mal. Específicamente en el caso del lactante, es probable que aún las mejores dietas y en el mejor ambiente no logren prevenir la carencia de hierro en todos los casos (15). La alternativa es la suplementación ya sea en forma de hierro medicamentoso o mediante el enriquecimiento de adimentos.

La suplementación con hierro medicamentoso por vía oral es poco practicable en gran escala, porque requiere de la administración diaria del medicamento por períodos prolongados de tiempo. Esto es difícil o imposible de lograr en pobla- 


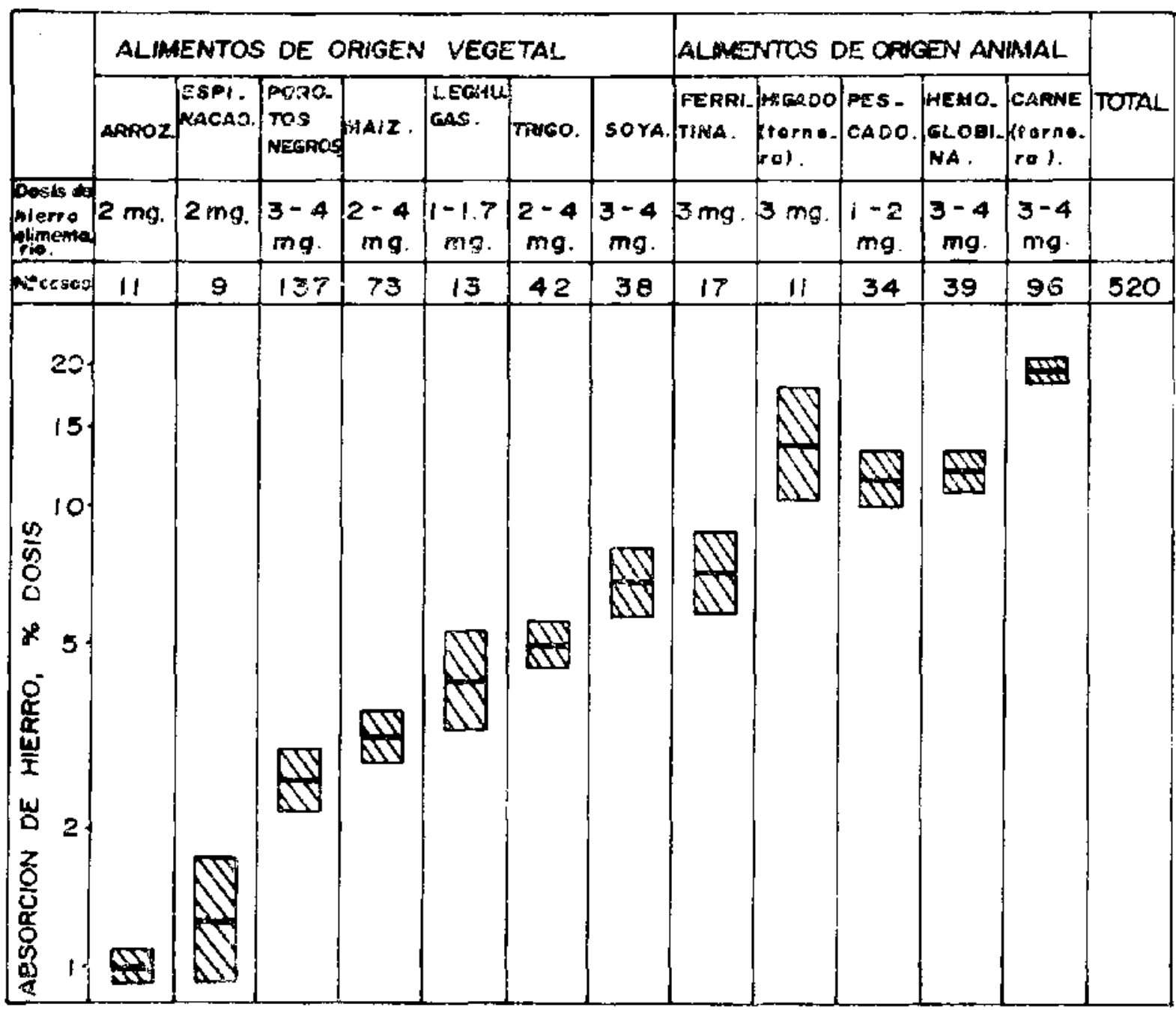

- Loyrissu, M. e e al. Picgr. Hemotal., 7 : 137, 1971.

ciones de bajo nivel cultural. La administración de preparados de hierro de depósito, además de requerir la vía parenteral, se ha visto asociada a un número considerable de reacciones secundarias por lo que no se presta para programas masivos de salud pública. Por estos motivos, grupos de expertos han concluido que el método más práctico para la eliminación de la deficiencia de hierro en una comunidad es probablemente el enriquecimiento de los alimentos con hierro. "Deben por lo tanto desarrollarse procedimientos adecuados de enriquecimiento prestando especial atención a la forma de hierro empleado, el alimento al cual se agrega, la cantidad de ese alimento consumido por los diferentes segmentos de la población, la adecuacidad de la absorción de hicrro de la dieta enriquecida, y la aceptabilidad del procedimiento por parte de los consumidores y de la industria alimenticia. La efectividad de cualquier programa de enriquecimiento debe ser evaluada en estudios de terreno controlados doble ciego que simulen las condiciones normales en la forma más aproximada posible”. (2). Se analizará cada uno de estos puntos.

La forma de hierro empleada debe ser una que se absorba en forma adecuada. Desgraciadamente, por desconocimiento o porque han prevalecido otras consideraciones tales como la de no modificar las características organolépticas del alimento enriquecido, este principio no siempre se ha tenido en cuenta. En los Estados Unidos, por ejemplo, se ha venido eentiqueciendo con hierro desde hace muchos años los cereales de uso infantil, sin que se note una disminución en la prevalencia de la 
deficiencia de hierro (15). Esto se debe probablemente a que la mayoría de estos cereales se han enriquecido con sales como el pirofosfato de sodio y hierro o el metofosfato de hierro que posteriormente se demostró que son muy pobremente absorbidos. En un estudio reciente de cuatro preparaciones empleadas para enriquecer pan (sulfato ferroso, hierro reducido de pequeño tamaño de partículas, ortofosfato férrico y pirofosfato de sodio $\mathrm{y}$ hierro) sólo el sulfato ferroso $\mathrm{y}$ el hierro reducido fueron absorbidos en forma adecuada (16). Esto enfatiza la necesidad de hacer estudios de absorción previos a las recomendaciones de enriquecimiento.

La cantidad y uniformidad con que son consumidos los alimentos susceptibles de enciquecimiento deben ser conocidas. El alimento seleccionado debiera ser consumido en la forma más universal y uniforme por el grupo expuesto, de manera de poder dosificar en la forma más precisa posible el hierro suplementario. El alimento a enriquecerse, además, debe ser procesado centralmente en algún momento ya que lógicamente no pueden ser enriquecidos alimentos que son producidos y consumidos a nivel local. Los aljmentos más frecuentemente usados para enriquecimiento han sido los cereales (trigo). Aunque en forma más reciente se han considerado otros como la sal y el azúcar.

En lactantes, los alimentos más susceptibles de enriquecerse son la Jeche y los cereales. El tipo, la cantidad y la forma de preparación de los cereales para niños varía ampliamente de un país a otro. En Chile, el consumo de estos productos (maicena, derivados de la harina de trigo) es muy elevado y serían susceptibles a enriquecerse, aunque las cantidades y los tipos consumidos son bastante variables (17). El consumo de leche, por otro lado, tiene necesariamente que ser más uniforme $\mathrm{y}$ aunque los tipos de leche pueden variar considerablemeente de un país a otro, puede afirmarse que grupos considerables de lactantes en la actualidad son alimentados con algún tipo de leche procesada, sea simplemente leche en polvo o bien fórmulas para lactantes especialmente preparadas.

La existencia en Chile desce hace varios años de programas masivos de entrega de leche en polvo a lactantes crea una situación particularmente apropiada para intentar el enriquecimiento de este producto. Nuestro grupo comenzó hace algunos años, estudios en este sentido siendo necesario inicialmente evaluar los efectos químicos y organolépticos del agregado de sales de hierro a leche en polvo en las condiciones locales de envase $y$ almacenamiento. Para todos los estudios se empleó leche semidescremada ( $12 \%$ de materia grasa) a la cual se agregó sulfato ferroso o citrato de hierro y amonio en una concentración de $10 \mathrm{mg}$. de hierro elemental por 100 gramos de polvo. Se evaluaron mezclas de leche y hierro con diversos antioxidantes (ácido ascórbico, d-toceferol y BHA) almacenadas en bolsas de polietileno por períodos de hasta 6 meses (3). La Tabla II resume los resultados de diversos tests que demostraron que bajo las condiciones descritas no habían efectos significativos del agregado de hierro sobre los caracteres de la leche. Sólo se detectó algún sabor

TABLA II

RESUMEN DE ENSAYOS DE CARACTERISTICAS ORGANOLEPTICAS (1) Y QUIMICAS EN LECHES SEMIDESCREMADAS ADICIONADAS CON HIERRO

\begin{tabular}{|c|c|c|c|c|c|c|c|c|}
\hline & Color & olor & $\begin{array}{l}\text { Sabor } \\
\text { polvo }\end{array}$ & $\begin{array}{l}\text { Sabor (3) } \\
\text { solucion } \\
\text { al 10\% }\end{array}$ & $\begin{array}{l}\text { Test del } \\
\text { TBA (4) }\end{array}$ & $\left|\begin{array}{c}\text { Test de } \\
\text { Pardea- } \\
\text { miento } \\
\text { no enzi- } \\
\text { mático }\end{array}\right|$ & Acidez & $\begin{array}{l}\text { Solubi- } \\
\text { lidad }\end{array}$ \\
\hline $\begin{array}{l}\text { Leche }+\mathrm{Fe} \mathrm{So} \\
\text { Leche }+\mathrm{Fe} \mathrm{So}_{4}+\text { ác. ascórbico } \\
\text { Leche }+\mathrm{Fe} \mathrm{So}+\alpha-\text { tocoferol } \\
\text { Leche }+\mathrm{Fe} \mathrm{So}_{4}+\mathrm{BHA}(2) \\
\text { Leche }+ \text { citrato } \mathrm{Fe} \text { y } \mathrm{NH}_{4}\end{array}$ & $\begin{array}{l}- \\
- \\
-\end{array}$ & $\begin{array}{l}- \\
- \\
-\end{array}$ & $\begin{array}{c}+ \\
+a++ \\
- \\
-a+ \\
-\end{array}$ & $\left\{\begin{array}{l}-a+ \\
+a++ \\
-a+ \\
-a+ \\
-\end{array}\right.$ & $\begin{array}{c}- \\
- \\
-\end{array}$ & $\begin{array}{l}- \\
- \\
-\end{array}$ & $\begin{array}{l}- \\
- \\
-\end{array}$ & $\begin{array}{l}- \\
- \\
-\end{array}$ \\
\hline
\end{tabular}

\footnotetext{
- Similar a leche testigo.

$+\quad=$ Ligera diferencia con leche testigo.

$++=$ Moderada diferencia con leche testigo.

$+++=$ Marcada diferencia con leche testigo.
}

(1) Características organolépticas determinadas por panel de 9 jueces. (Gentileza CbiprodaI S. A. I., Stgo).

(2) $\mathrm{BHA}=$ butil-hidroxi-anisol.

(3) Nota: Las diferencias de sabor se refieren a sabores ácido y metálico. No se encontró rancidez.

(4) TBA $=$ ácido tiobarbitúrico. 
metálico en las leches con sulfato ferroso pero en ningún caso rancidez. El bajo contenido graso de la leche utilizada posiblemente ayudó en este sentido, ya que el hierro favorece la aparición de rancidez al acelerar la oxidación de la grasa. Este tipo de estudios eran importantes porque si bien existen en el mercado leches enriquecidas, como el Similac con Hierro, (R), se trata de productos altamente elaborados (con reemplazo de la grasa animal por aceites vegetales) y muy bien envasados (evitándose la acción oxidante del contacto con $\mathrm{cl}$ aire). Estudios posteriores no han revelado combios discernibles en la calidad de leches en polvo semidescremadas adicionadas con $15 \mathrm{mg}$. de hierro por 100 gramos (como sulfato ferroso) y almacenadas en condiciones de terreno por períodos de hasta 13 meses.

A pesar de que en algunos países existen en el mercado leches enriquecidas con hierro desde hace considerable tiempo, existen sorprendentemente pocos estudios de absorción del hierro administrado en este vehículo. Hace ya varios años, Shulz y Smith $(18,19)$ comunicaron un promedio aritmético de absorción de $9,1 \%$ del hierro natural de la leche en 10 niños normales empleando la técnica de incorporación biológica a la leche de $\mathrm{Fe}^{59}$. En 4 lactantes deficieentes en hierro la obsorción promedio fue de $16 \%$. Estos autores estudiaron luego la absorción de una dosis alta de hierro suplementario $(30 \mathrm{mg}$. de hierro como sulfato ferroso administrado con $180 \mathrm{ml}$, de leche entera homogenizada) en 6 niños normales y 2 niños deficientes obteniendo promedios de absorción de $6 \%$ y $13 \%$ respectivamente.

Nuestro grupo hà comunicado recientemente los resultadós de estudjos de absorción de hierro agregado a leche en 110 lactantes empleando métodos isotópicos (20). Se emplearon leches adicionadas con $10 \mathrm{mg}$. de Fe por litro (o $100 \mathrm{gm}$. poivo) en forma de sulfato ferroso. Se administró a cada lactante una mamadera en ayunas, oscilando la dosis ingerida entre 1,0 y 2,8 de $\mathrm{Fe}(100$ a $280 \mathrm{~mL}$. de leche). Todos los estudios fueron realizados en lactantes sanos entre 6 y 18 meses de edad. Los promedios de absorción comunicados originalmente fueron de $6,9 \%$ para leche semidescremada, $7,1 \%$ para leche semidescremada más maicena, $12,5 \%$ para leche semidescremada más ácido ascórbico y $3,5 \%$ para leche entera. En comparación, la absorción de una solución acuosa de ascorbato de hierro fue mucho más alta (Tabla III). Cabe hacer notar sin embargo, que el rango de valores de absorción fue muy amplio como es característico en este tipo de estudios. Además la distribución de valores no siguió uua curva normal sino que se observó la característica asimetría con acumulación de sujetos en los rangos bajos de absorción. Este fenómeno ha sido analizado en detalle por Cook y cols. (21). Estos autores han concluido que la transformación logarítmica de los valores permite normalizar la distribución y los promedios geométricos asi obtenidos son mucho más representativos que los promedios aritméticos. Al calcular la absorción en es-

TA B LA III

ABSORCION DE HIERRO AGREGADO A LA LECHE. PROMEDIO Y RANGO DE LAS OBSERVACIONES EN 110 LATANTES

Absorción de Hierro (\%)

\begin{tabular}{|c|c|c|c|c|c|c|c|c|}
\hline & $\begin{array}{l}\text { Peso } \\
(K g)\end{array}$ & $\begin{array}{c}\text { Hemo- } \\
\text { blobina } \\
\text { (g\%) }\end{array}$ & $\begin{array}{l}\text { Hierro } \\
\text { Sérico } \\
\text { (ug\%) }\end{array}$ & $\begin{array}{c}\text { Satura- } \\
\text { cibn de la } \\
\text { transfe- } \\
\text { rrina }(\%)\end{array}$ & $\begin{array}{c}\text { Dosis de } \\
\text { Hierro } \\
\text { (mg) }\end{array}$ & Rango & $\mid \begin{array}{c}\text { Prome- } \\
\text { dio Arit- } \\
\text { mético }\end{array}$ & $\begin{array}{l}\text { Prome- } \\
\text { dio Geo- } \\
\text { mérrico }\end{array}$ \\
\hline Leche semidescremada & 9,4 & 11,8 & 59 & 14,1 & 1,5 & & 6,9 & 3,7 \\
\hline $\begin{array}{l}\text { (35 lactantes) } \\
\text { Leche semidescremada }\end{array}$ & $7,2 \cdot 11,9$ & $7,0-14,5$ & $16-140$ & $3,8-29,6$ & $1,0-2,1$ & $0,2-26,5$ & & \\
\hline+ maicena & 9,2 & 11,6 & 57 & 14.2 & 2,0 & & 7,1 & 4,7 \\
\hline (17 Jactantes) & $7,4-10,9$ & $9,0-13,9$ & $22-136$ & $5,2-28,3$ & $1,4-2,6$ & $0,3-18,1$ & & \\
\hline Leche semidescremada & 00 & & & & & & & \\
\hline + ácido ascórbico & 8,9 & 11,9 & 48 & 12,0 & 1,6 & & 12,5 & 5,9 \\
\hline (16 lactantes) & $6,3-12,8$ & $10,2-13,7$ & $18-98$ & $4,1-24,7$ & $1,1-2,4$ & $0,3-41,1$ & & \\
\hline Leche entera & 8,7 & 11,8 & 64 & 18,1 & 1,8 & & 3,5 & 2,1 \\
\hline (19 lactantes) & $7,0-11,6$ & $9,8-13,2$ & $37-94$ & $10,3-32,6$ & $1,1-2,8$ & $0,5-13,4$ & & \\
\hline Ascorbato de hierro & 8,2 & 11,7 & 50 & 14,2 & 2,2 & & 23,4 & 18,2 \\
\hline (23 lactantes) & $6,2-11,6$ & $8,0-13,7$ & 24.96 & $6,5-24,7$ & $1,8-3,1$ & $2,6-61,6$ & & \\
\hline
\end{tabular}


ta forma en nuestros sujetos se obtienen promedios considerablemente más bajos que los comunicados originalmente (Tabla III), del orden del $4 \%$ para la leche semidescremada sola o con maicena y cercana al $6 \%$ en la leche, más vitamina $C$.

Mediante el empleo de leches enriquecidas, varios autores en EE. UU. han comunicado efectos favorables sobre el estado de nutrición de hierro en estudios de terreno $(22,10)$. Efectos favorables también se han obtenido en Suecia mediante el uso de cereales enriquecidos (23). En el estudio de Andelman y Sered por ejemplo, en que se empleó leche enriquecida desde el nacimiento los resultados fueron sorprendentemente favorables. En grupos grandes de lactantes de muy bajo nivel socioeconómico observados durante 18 meses, un $76 \%$ de los lactantes alimentados con leche no enriquecida desarrolló anemia en tanto que se observó solamente un $9 \%$ de anemia en el grupo suplementado (10).

Con estos antecedentes y nuestros datos previos de absorción comenzamos en Santiago un estudio de terreno longitudinal con leche semidescremada enriquecida. El producto que estamos empleando contiene $15 \mathrm{mg}$. de hierro (como sulfato ferroso) por $100 \mathrm{gm}$. de polvo. En este estudio la suplementación comienza a los 3 meses de edad y dura un año, entregándose $3 \mathrm{~kg}$. mensuales de leche enriquecida los primeros 6 meses y $2 \mathrm{~kg}$. al mes Ios últimos 6 meses. La aceptabilidad y tolerancia de la leche con hierro han sido excelentes. Se pueden calcular con este programa ingestas diarias de 10 a $15 \mathrm{mg}$. de hierro suplementario, cifras que estarían incluso por encima de las recomendaciones. Sin embargo, si en la práctica el hierro se absorbe con el promedio determinado por nosotros de sólo $4 \%$, se estarían absorbiendo 0,4 a $0,6 \mathrm{mg}$. al día, o sea, el $50 \%$ de la recomendación de $1 \mathrm{mg}$. de hierro absorbido al día (o el $70 \%$ de recomendación mâs reciente de $0,7 \mathrm{mg} /$ g. de hierro absorbido sobre los 5 y 12 meses).

Aunque la deficiencia de hierro en preescolares es menos prevalente (24) que en el lactante, también este grupo requiere suplementación. En general, el enriquecimiento de alimentos para consumo de estos niños ofrece problemas similares a los que se encuentran en adultos. El alimento a enriquecerse dependerá estrictamente de las consideraciones locales y deberán tomarse en cuenta los conceptos sobre absorción de hierro unido a alimentos ya analizados. Se dijo que el hierro de alimentos vegetales o suplementando dietas predominantemente vegetales se absorbe en forma pobre. En este sentido, se han emitido opiniones pesimistas (14) sobre las posibilidades de entiquecimiento de dietas vegetales ya que es posible que aún en cantidades elevadas de hierro en dietas carentes de proteínas animales no logren conseguirse absorciones adecuadas. Existe, sin embar- go, un número creciente de alimentos de alto contenido proteico y bajo costo que se están desarrollando específicamente para el grupo de preescolares y cuyo consumo seguramente aumentará en el futuro. Estos alimentos, al ser procesados centralmente, constituirían un vehículo muy apropiado para hierro suplementario. Algunos de estos productos que han alcanzado producción en gran escala son Cerealinea, producido en Brasil y compuesto de harina de soya, almidón de maíz y leche en polvo: Incaparina, una mezcla de cereales producida en Guatemala compuesta de harinas de semilla de algodón y de maíz y tórula; Vitalia, un producto tipo macaroni producido en Colombia en base a semolina de trigo y derivados de soya y arroz y Fortesan, producido en Chile con una mezcla de trigo, soya y leche. La mayoría de estos productos contienen una cantidad adicional de minerales y vitaminas. El contenido de hierro de estos productos y las posibilidades de enriquecimiento debieran ser estudiados.

\section{REFERENCIAS}

1.-Joint FAO/WHO. Expert Group on Requirements of Ascorbic Acid, Vitamin D, Vitamina $B_{12}$, Folate and Iron. Wld. Hlth. Org. techn. Rep. Ser. No 452, 1970.

2.-WHO Ssientific Group on Nutritional Anaemias. Hith. Org. techn. Rep. Ser., No 405, 1968.

3.--Mandujano, Y., Vergara, L. y Stekel, A. Carencia de Hierro en el Lactante. Tesis, Fac. Quim. Farm., U. de Chile, 1970.

4.- Margozzint, J., Bravo, M., y cols. La carencia de Fierro en el Lactante considerado Eutrófico Area Hospitalaria Norte de Santiago. Rev. Chil. Pediat. 43: 9, 1972.

5.-Winter, A., Taboada, H, y cols. Incidencia de Anenia y Ferropenia en el Lactante Chileno. IX Reunión Anual Soc. Lat. Iny. Ped. Valdivia, Chile, 1969.

6.-Eiwood, P. C. Some Epidemiological Aspects of Iron Deficiency Relevant to its Evaluation: Proc. Roy. Soc. Med. 63: 1230, 1970.

7.-Howell, D. Clinical Problems of Iron Deficiency in Early Life. Sixty Second Ross Conference on Pediatric Research, Iron Nutrition in Infancy, 1970.

8.-W Wib, T.E. and Oski F. A. Iron Deficiency Anemia and Scholattic Achievement in Young Ado lescents. J. Pediat., 82: 827, 1973. 
9.-Jacobs, A., Bentley, D. P., Joynson, D. and Jones, $P$. Inmunological Abnormalities in Iron Deficiency Anemia. Meeting Brit. Soc. Hematol., Aberdeen, 1973.

10.-Andelman, M. B. and Sered, B. R. Utilization of Dietary Iron by Term Infants. Amer. J. Dis. Child. 111: $54,1966$.

11.-Bothwell, T. H., and Finch, C. A. Iron Metabolism. Little Brown, Boston, 1962.

12.-Moore, C. V. Iron Nutrition, in Iron Metabolism; International Symposium. Springer, Berlin, 1964.

13.- Layrisse, M., Cook, I. D., Marinez, C. Roche, $M$, Kahn, 1. N., Walker, R. B. and Finch, C. A. Iron Absorption: A Comparison of Vegetable and Animal Foods. Blood. 33: 430, 1969.

14.-Layrisse, M. and Martinez-Torres, C. Food Iron Absorption: Iron Supplementation of Food. Progr. Hematol., 7: 137, 1971.

15.-Committee on Nutrition: Iron Balance and Requirements in Infancy. Pediatrics, 43: 134, 1969.

16.-Cook, J. D., Minnich, V., Moore, C. V. Rasmussen, A, Bradley, W. B., and Finch, C. A. Absorption of Fortification Iron in Braed. Am. J. Clin. Nutr., 26: 861, 1973.
17.-Minte, P. Estudio de Mcreadeo de los Alimentos Infantiles en Cbile. Informe Esc. Administración, Univ. de Chile, 1972.

18.- Shulz, $J$. and Smith, N. J. A Quantitative Sludy of the Absorption of Food Iron in Infants and Children. Amer. J. Diș, Child., 95: 109, 1958.

19.-Shalz, J. and Smith, N. J. Quantitative Study of the Absorption of Iron Salts in Infants and ChilIren. Amer. J. Dis. Child, 95: 120, 1958.

20.-Stekel, A., Olivares, M. and López, I. Absorption of Iron Added to Milk in Infants (por publicarsc), IX Congr. Int. de Nutr,, México, 1972.

21.-Cook. J. D., Layrisse, M. and Finch, C. A. The Measurement of Iron Absorption. Blood. 33: 421, 1969.

22.-Marsh, A., Loug, H. and Stierwalt, E. Comparative Hematologic Response to Iron Fortification of a Milk Formula for Infants. Pediatrics. 24: 404, 1959.

23,-Moe, $P$. J. Iron Requirements in Infancy. Acta Paediat., suppl. 150, 1963.

24.-Amar, M., Muñoz, I., y Siekel, A. Ingesta y Carencia de Hierro en Pre-Escolares Chilenos. (Por publicarse). 